\title{
Multiple dual-wavelengths fiber ring laser utilizing a phase-only sampled fiber Bragg grating with multiple phase-shifts inserted
}

Xuxing Chen, Takasi Kameyama, Ming Li, and Hongpu Li*

Shizuoka University, Johoku 3-5-1, Hamamatsu 432-8561, Japan, *dhli@ipc.shizuoka.ac.jp, Tel. /Fax: +81-53-4781617

\begin{abstract}
A simple technique to multiply the lasing wavelength in a semiconductor optical amplifier (SOA)-based fiber ring laser is proposed and demonstrated, which is based on the utilization of a multiple phase-shifts sampled fiber Bragg grating(FBG). Tunability for the wavelength spacing within each dual-wavelengths has also been demonstrated by changing the positions of phase shifts. Moreover, more than 20 dual-wavelengths fiber lasers with a 3-dB linewidth of $0.017 \mathrm{~nm}$ and signal-to-noise ratio (SNR) of about $45 \mathrm{~dB}$ have been obtained.
\end{abstract}

Key words: Multiwavelength fiber laser; notch filter; phase-shifted fiber Bragg grating;

PACS: $\quad$ 42.55.Wd, 42.79.Ci, 42.81.Wg 


\section{Introduction}

Multi-wavelength fiber lasers have attracted great interest due to their applications to optical communication, optical fiber sensing, optical component testing, and microwave photonics [1-18]. To date, various kinds of multiwavelength fiber lasers have been proposed and demonstrated, however multiple dual-wavelength fiber lasers have rarely been demonstrated except for the one reported in Ref. [13], where the Erbium-doped fiber (EDF) was used as a gain medium and a narrowband polarization-maintaining (PM) Fabry-Pérot filter (FPF) was utilized and as a result, the power ratio of the dual-wavelengths can be switched by adjusting the polarization controller (PC). However, the wavelength spacing between the dual wavelengths in that case was fixed. Moreover, a considerably high injected power is strongly desired to reduce the cross gain saturation in the EDF by introducing the nonlinear polarization rotation effect in the fiber ring, which may not be easily realized [13-14].

Most recently, we have proposed a technique for the realization of a multi-channel notch filter by using a thermally-induced phase shifted phase-only sampled FBG [19-21]. Both the single-phase and multiple-phases shifted FBGs were investigated in details. We theoretically and experimentally demonstrated that the channel count of the notch filters could be multiplied by thermally introducing multiple $\pi$ phase-shifts. In this letter, based on utilization of this kind of FBG, we demonstrate a novel semiconductor optical amplifier (SOA)-based fiber ring laser. The key feature of this fiber laser lies in its strong flexibilities of multiplication of the lasing wavelengths and the tunability for the wavelength spacing between each dual-wavelengths. Moreover, thanks to the utilization of the sampled multichannel FBG, the obtained fiber laser has a 
fixed wavelength spacing (0.8 $\mathrm{nm}$ in this paper) between each pair of the dual-wavelength, which is exactly compatible with the standard of international telecommunications union (ITU) grids.

\section{Experimental Setup}

Figure 1 shows the schematic diagram of the experimental setup for the multiwavelength fiber laser. This setup consists of a semiconductor optical amplifier (SOA: with a maximum small-signal gain of $19 \mathrm{~dB}$ and a polarization dependent gain of $0.7 \mathrm{~dB}$ ), two isolators, a polarization controller (PC), a 10/90 power coupler, a circulator, and two concatenated 51-channel phase-only sampled FBGs, which have identical characteristics with a channel bandwidth (1dB) of $0.6 \mathrm{~nm}$ and channel spacing $0.8 \mathrm{~nm}$ [19]. The length of the ring cavity is about $35 \mathrm{~m}$, producing a cavity mode spacing of $5.8 \mathrm{MHz}$, and the total loss of the cavity is estimated to be approximately 11 $\mathrm{dB}$. The two isolators are used to guarantee the unidirectional optical propagation and the PC is used for the stabilization of the laser output $[15,17]$. The inset of Fig. 1 shows schematic diagram of the multiple-phases shifted phase-only sampled FBG. Several $\mathrm{NiCr}$ wires (heaters) fixed in the V-grooves are employed to induce multiple phase-shifts in the FBG based on thermal-optical effect. In particular, three NiCr wires labeled as \#1, \#2 and \#3 are employed in our setup. By switching on any two of the driving circuits simultaneously and tuning the corresponding variable resistors, two $\pi$ phase-shifts can be inserted at the grating positions where the $\mathrm{NiCr}$ wires are located. Moreover, the separation between these two phase-shifts could be changed in steps either by heating different two NiCr wires or by changing the separation of each of the 
two wires mechanically. To keep the thermal stability, a heat sink made from a thin copper plank is placed over the crossing area, meanwhile a small fan attached to the utilized heat sink is employed to dissipate the unwanted heat conduction which partly determines the stability of this fiber laser.

\section{Experimental Results and Discussions}

Once two $\pi$ phase shifts are simultaneously introduced into the phase-only sampled FBG, a 51-channel dual-bandpass filter can be generated in the transmission spectrum of the two concatenated FBGs as is shown in Fig. 2(a). The unequal transmission within each channel at longer wavelength may be due to either the thermal induced birefringence of the dispersion slope of the FBG [20]. For easily seen, the spectrum covering only the central three channels in Fig. 2(a) is illustrated in Fig. 2(b). It can be seen that a narrow dual-bandpass filter with two bandwidths of $0.028 \mathrm{~nm}$ and $0.027 \mathrm{~nm}$ (i.e., full width at half maximum: FWHM) has been obtained. In a certain extent, this narrow bandwidth will ultimately determine the linewidth of the SOA-based multiwavelength laser in our case. Figure 3(a) shows the output spectrum of the proposed fiber laser working at room temperature. It is seen that more than 20 dual-wavelength lasing with the channel spacing of $0.8 \mathrm{~nm}$ has been obtained. Note that, due to the birefringence probably existing in the SOA and the phase-shifted FBG, the number of the lasing wavelengths and the spectral shape of the multi-wavelength output are sensitive to the polarization controller. By carefully adjusting the polarization in the cavity, we obtain the maximum number of the stable lasing wavelength, which is limited by the bandwidth of the gain spectrum of the utilized SOA. To further increase the number of the lasing wavelengths and produce the uniform output power spectrum, 
it should be possible to utilize a second SOA with a slightly offset gain spectrum to the first one [22]. Fig. 3(b) shows the spectrum of one typical dual-wavelength lasing in Fig. 3(a). The signal-to-noise ratio (SNR) and the 3-dB linewidth of the dual-wavelength lasing are higher than $45 \mathrm{~dB}$, and less than $0.017 \mathrm{~nm}$, respectively, which are very difficult to be realized in the general SOA based fiber ring laser [14]. Moreover, since the phase-only sampled FBG in our experiment is particularly designed and fabricated as a simultaneous dispersion and dispersion-slope compensator in practical optical communication system [20], the obtained fiber laser has a fixed spacing of $0.8 \mathrm{~nm}$ between each dual-wavelength which makes it exactly compatible with the standard of ITU and thus easier to be integrated into the commercial WDM system.

To demonstrate the switching ability of the fiber laser between the normal single-wavelength and multiple dual-wavelengths lasing, only one $\pi$ phase shift is introduced into the FBG. Fig. 4(a) shows one typical channel of the multi-channel narrow bandpass filter with only one $\pi$ phase shift inserted, and the output spectrum of typical single-wavelength lasing is illustrated in Fig. 4(b). Since there exists no interferences between multiple phase-shifts, the FWHM of bandpass spectrum with only one introduced $\pi$ phase shift becomes $0.025 \mathrm{~nm}$ which is smaller than the one (i.e., $0.028 \mathrm{~nm}$ ) of multiple $\pi$ phase-shifts. Accordingly, the output spectrum of fiber laser exhibits a narrower linewidth $(0.013 \mathrm{~nm})$ and a higher SNR (50 dB).

Next, to investigate the spectral stability of the proposed laser, we repeatedly measure the output spectrum of one typical dual-wavelength lasing 7 times in every other 5 minute by using an optical spectrum analyzer. Figure 5(a) shows the seven-scanned output power spectra of the typical dual-wavelength lasing. The maximum variation of the peak output power for each lasing is less than $0.8 \mathrm{~dB}$ and the 
maximum wavelength fluctuation is less than $0.01 \mathrm{~nm}$, which shows a good stability of the laser. The power and wavelength fluctuations may be attributed to the unstability of heats dispersion of the two NiCr wires. Figure 5(b) shows the output spectra of the two typical dual-wavelength lasing, which correspond to the cases where the \#1\&\#3 $\mathrm{NiCr}$ wires (the separation is $3.5 \mathrm{~cm}$ ) and \#2\&\#3 NiCr wires (the separation is $2.2 \mathrm{~cm}$ ) are heated, respectively. Since the length of the linearly chirped phase-only sampled FBG used is $12 \mathrm{~cm}$ and the bandwidth of each channel is $0.6 \mathrm{~nm}$, a separation of $3.5 \mathrm{~cm}$ and $2.2 \mathrm{~cm}$ for the $\pi$ phase shifts inserted will be accordingly related to a wavelength interval of $0.175 \mathrm{~nm}$, and $0.11 \mathrm{~nm}$, respectively. The wavelength spacing within the dual-wavelength lasing shown in Fig. 5(b) are about $0.178 \mathrm{~nm}$ and $0.116 \mathrm{~nm}$, respectively, which agree well with what we expect. The results shown in Fig. 5(b) mean that wavelength spacing within each dual-wavelength may also be tunable in our case. It is expected that a multiple triplex- or quadruple-wavelength fiber SOA-fiber ring laser can also be implemented if more discrete phase shifts are introduced.

\section{Conclusions}

In conclusions, a novel approach to multiply the lasing wavelength of semiconductor optical amplifier (SOA)-based fiber ring laser is experimentally demonstrated. More than 20 dual-wavelengths fiber lasers have been obtained with a 3-dB linewidth and signal-to-noise ratio (SNR) of about $0.017 \mathrm{~nm}$ and $45 \mathrm{~dB}$, respectively. Tunability for the wavelength spacing within each dual-wavelengths has also been realized by simply changing the positions of the phase shifts. Moreover, the obtained fiber laser has a fixed spacing of $0.8 \mathrm{~nm}$ between each dual-wavelength, which makes it exactly compatible 
with the standard of ITU and thus easier to be integrated into the commercial WDM system. It is expected to find the its potential application to coherent optical communication, fiber optic sensing, spectroscopy, and optical components testing systems.

\section{Acknowledgement}

This work was partly supported by the International Communications Foundation and Hamamatsu Science and Technology Promotion in Japan.

\section{References}

1. J. Chow, G. Town, B. Eggleton, M. Ibsen, K. Sugden, and I. Benion, IEEE Photon. Technol. Lett. 8, 60 (1996).

2. L. Talaverano, S. Abad, S. Jarabo, and M. Lopez-Amo, IEEE J. Lightwave. Technol. 19, 553 (2001).

3. X. Feng, C. Lu, H. Y. Tam, and P. K. A. Wai, IEEE Photon. Technol. Lett. 19, 1334 (2007).

4. S. Yamashita, and K. Hotate, Electron. Lett. 32, 1298 (1996).

5. A. Bellemare, M. Karasek, M. Rochette, S. LaRochelle, and M. Tetu, IEEE J. Lightwave Technol. 18, 825 (2000).

6. A. J. Poustie, N. Finlayson, and P. Harper, Opt. Lett. 19, 716 (1994).

7. M. Ibsen, E. Ronnekleiv, G. J. Cowle, M. N. Zervas, and R. I. Laming, Electron. Lett. 36, 143 (2000).

8. G. J. Chow and D. Y. Stepanov, IEEE Photon. Technol. Lett. 8, 1465-(1996).

9. S. Yamashita and Y. Inoue, Jpn. J. Appl. Phys. 44, 1080 (2005).

10. S. Pan, C. Lou, and Y. Gao, Opt. Express 14, 1113 (2006). 
11. X. Fang, H. Y. Tan, and P. K. A. Wai, Opt. Express 14, 8205 (2006).

12. R. Slavik, I. Castonguay, S. LaRochelle, and S. Doucet, IEEE Photon. Technol. Lett. 16, 1017 (2004).

13.G. X. Ning, J. Q. Zhou, S. Aditya, P. Shum, V. Wong, D. Lim, IEEE Photon. Technol. Lett. 20, 1606 (2008).

14. Z. Chen, S. Ma, and N. K. Dutta, Opt. Express 17, 1234 (2009).

15. H. Chen, Opt. Lett. 30, 619 (2005).

16. J. Yao, J. P. Yao, Z. Deng, and J. Liu, IEEE/OSA J. Lightwave Technol. 23, 2484 (2005).

17. Y. Han, F. Fresi, L. Poti, J. H. Lee, and X. Dong, Opt. Lett. 32, 1032 (2007).

18. X. Chen, J. Yao, and Z. Deng, Opt. Lett. 30, 2068 (2005)

19. M. Li, H. Li, and Y. Painchaud, Opt. Express 16, 19388 (2008).

20. M. Li and H. Li, Opt. Express 16, 9821 (2008).

21. H. Li, M. Li, Y. Sheng, and J. E. Rothenberg, IEEE J. Lightwave Technol. 25, 2739 (2007).

22. D. Moon, B. Kim, A. Lin, G. Sun, W. Han, Y. Han, and Y. Chung, Opt. Express 15, 8371(2007). 


\section{List of figure captions}

Fig. 1 Experimental setup for lasing wavelength multiplication of SOA-fiber ring laser, the inset llustrates the schematic diagram of the multiple-phases shifted phase-only sampled FBG.

Fig. 2 (a) Measuring results of the multi-channel narrow dual-bandpass filter as two $\pi$ phase-shifts are introduced, and (b) the central three channels of the dual-bandpass spectrum in Fig. 2(a).

Fig. 3. (a) Output spectrum of the SOA-based multiple dual-wavelength SOA-fiber ring laser, and (b) spectrum of Fig. 3(a) for typical dual-wavelength lasing.

Fig. 4 (a) Measuring results of the multi-channel narrow bandpass filter as only one $\pi$ phase shift is introduced, and (b) output spectrum of typical single-wavelength lasing with one $\pi$ phase shift.

Fig. 5 (a) Seven output power spectra of typical dual-wavelength lasing with a time interval of 5 minutes, and (b) output spectra of the typical dual-wavelength lasing with two different spacing $0.178 \mathrm{~nm}$ and $0.116 \mathrm{~nm}$, respectively. 


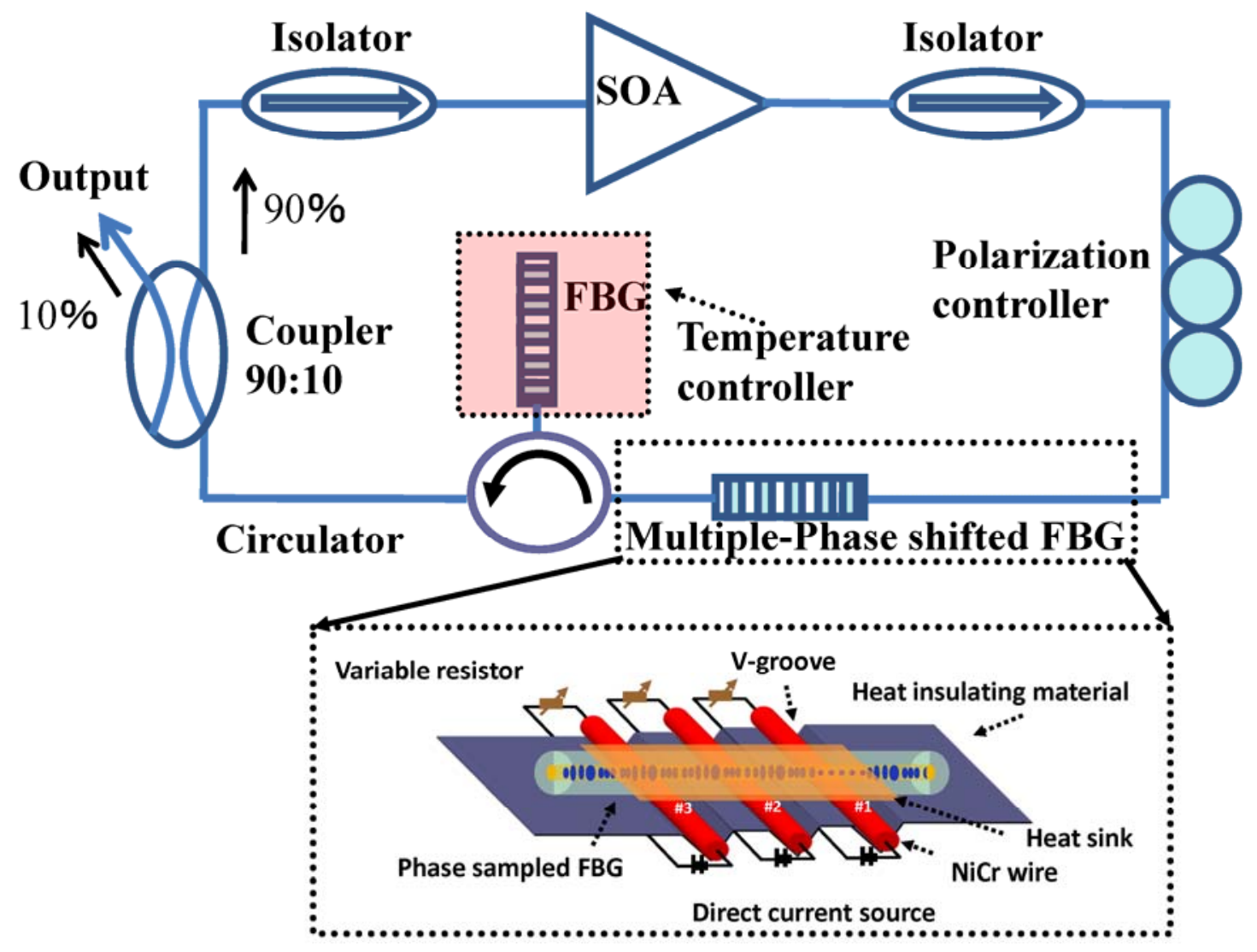

Fig. 1 Experimental setup for lasing wavelength multiplication of SOA-fiber ring laser, the inset illustrates the schematic diagram of the multiple-phases shifted phase-only sampled FBG. 

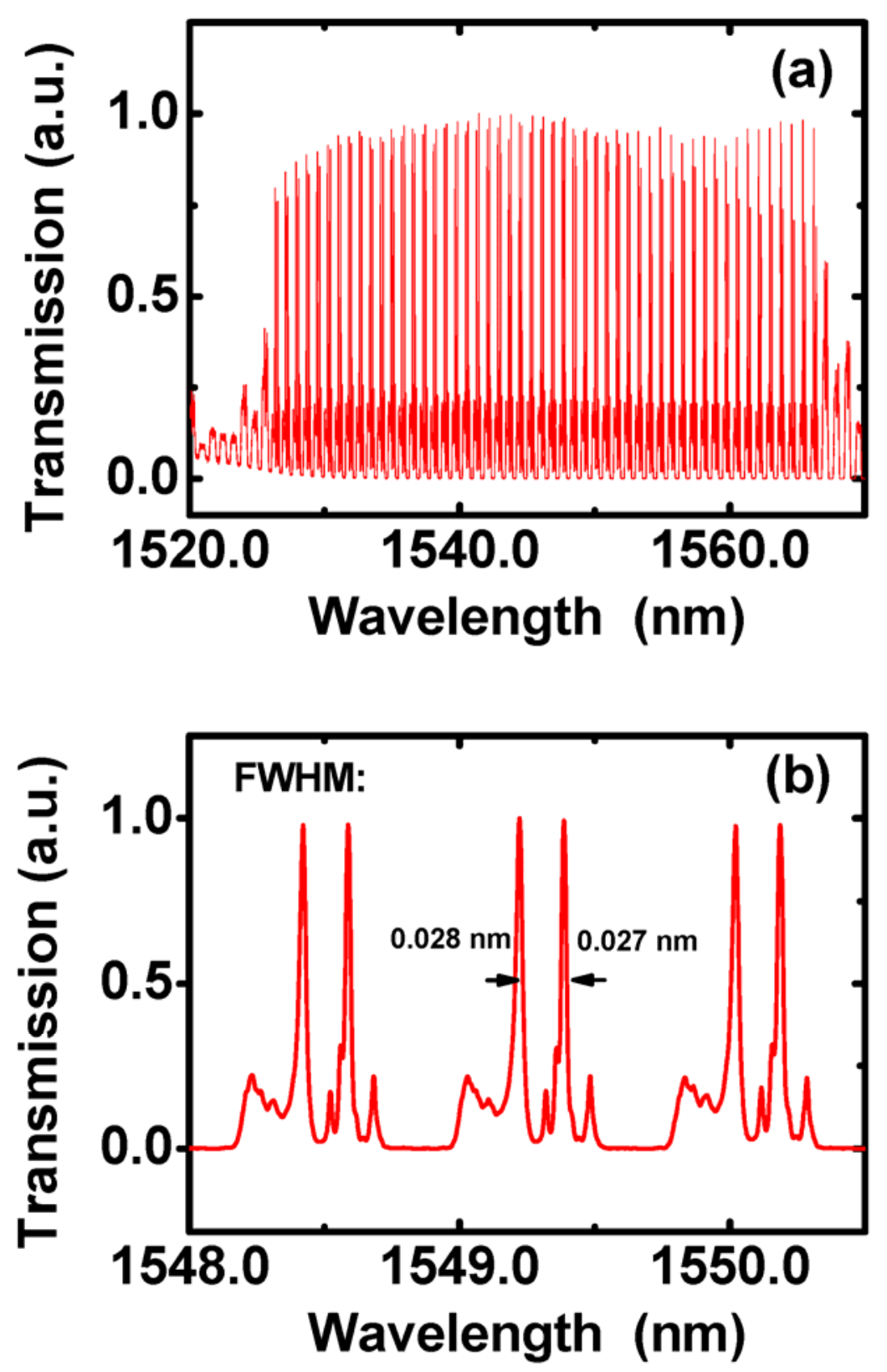

Fig. 2 (a) Measuring results of the multi-channel narrow dual-bandpass filter as two $\pi$ phase-shifts are introduced, and (b) the central three channels of the dual-bandpass spectrum in Fig. 2(a). 

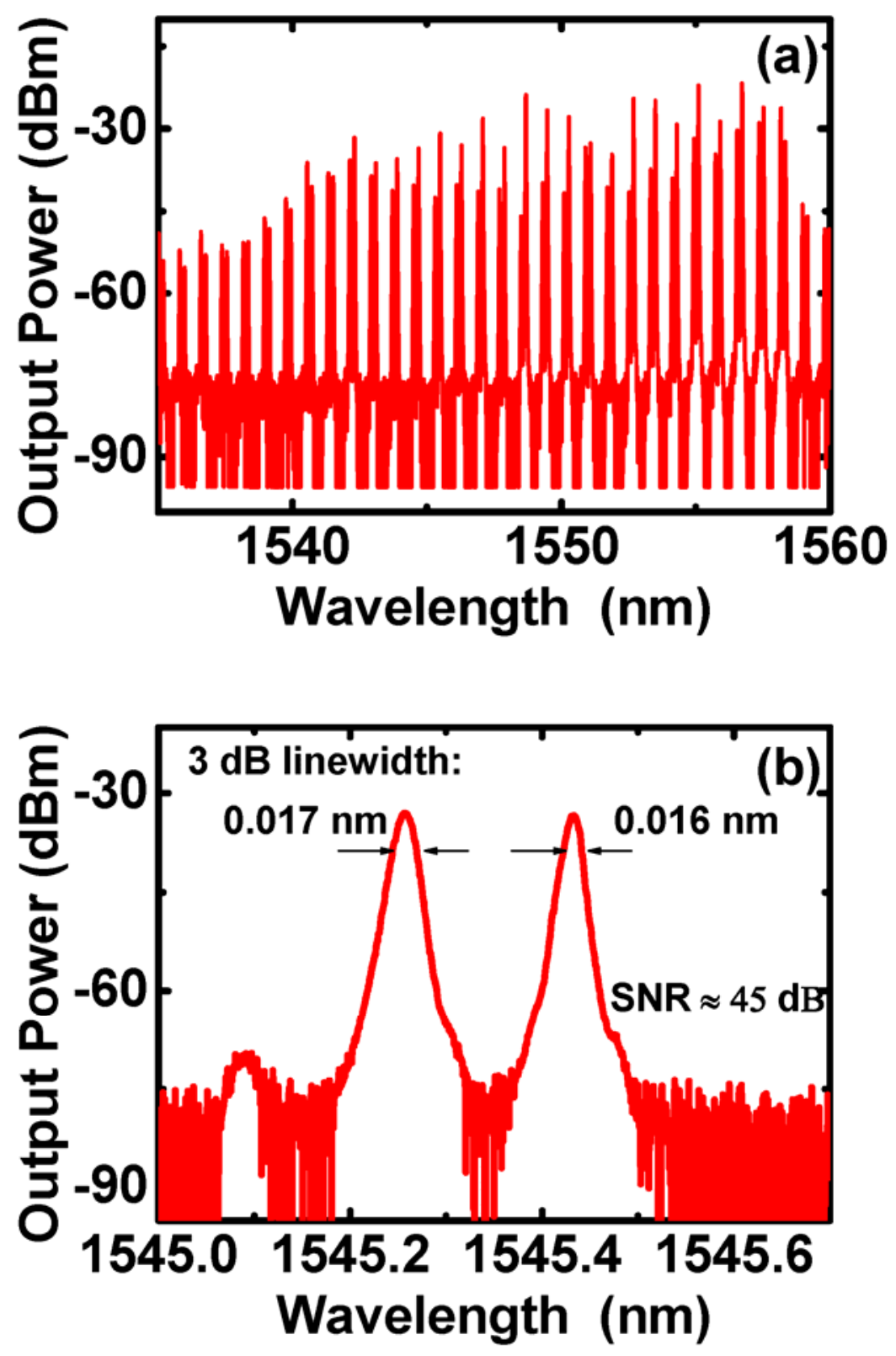

Fig. 3 (a) Output spectrum of the SOA-based multiple dual-wavelength SOA-fiber ring laser, and (b) spectrum of Fig. 3(a) for typical dual-wavelength lasing. 

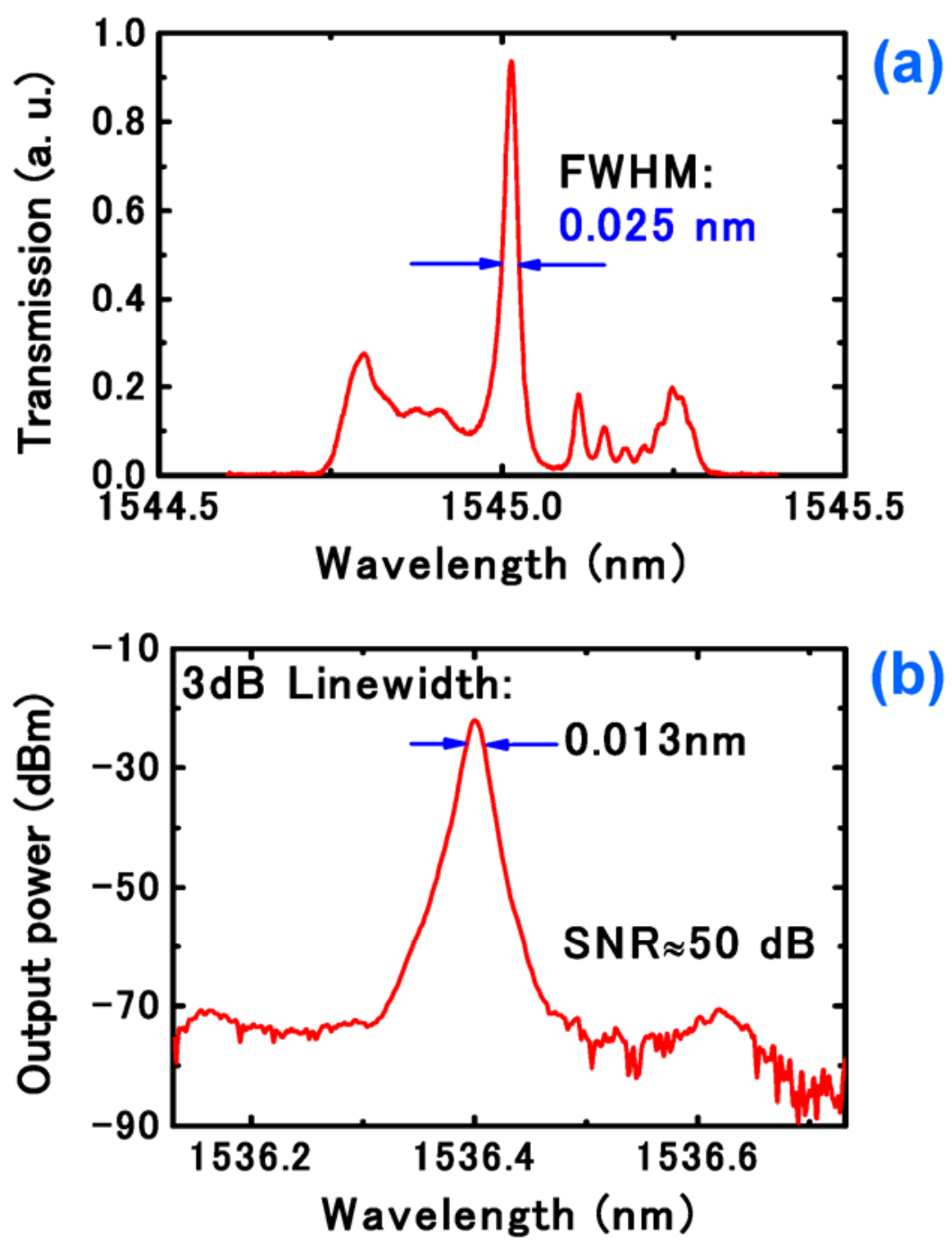

(b)

Fig. 4 (a) Measuring results of the multi-channel narrow bandpass filter as only one $\pi$ phase shift is introduced, and (b) output spectrum of typical single-wavelength lasing with one $\pi$ phase shift. 

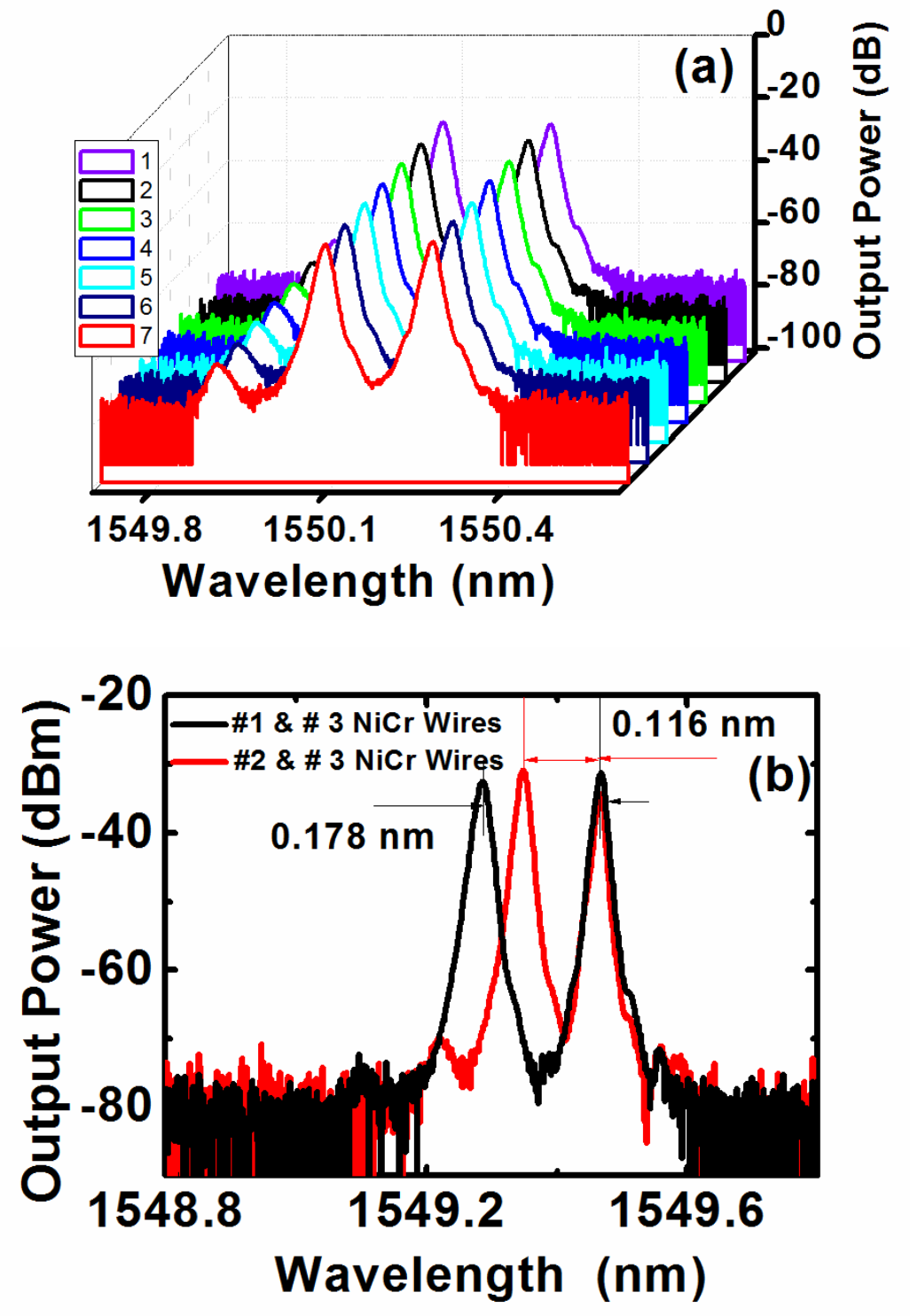

Fig. 5 (a) Seven output power spectra of typical dual-wavelength lasing with a time interval of 5 minutes, and (b) output spectra of the typical dual-wavelength lasing with two different spacing $0.178 \mathrm{~nm}$ and $0.116 \mathrm{~nm}$, respectively. 